\title{
Peter Waterhouses literarische Verhandlungen der Flucht und Asylsuche: Die Auswandernden (2016)
}

\begin{abstract}
The article focuses on the prose experiment Die Auswandernden by the Austrian writer Peter Waterhouse and the illustrator Nanne Meyer in 2016. The book falls in the category of literary texts reflecting refugee and asylum-seeker experiences ${ }^{1}$ that have appeared on the German book market since 2012. The paper aims to describe the literary strategies Waterhouse uses to represent the refugee condition. It explores the author's implicit programmatic statements, the construction of the female protagonist as a refugee and asylum seeking figure and Waterhouse's reflections on language, especially the language of Austrian judiciary system.
\end{abstract}

Keywords: Peter Waterhouse, Nanne Meyer, refugee experience, contemporary Austrian literature, language reflection, reflection on language of the judicial system, experimental fiction

\section{Problematyka migracji i poszukiwania azylu w Die Auswandernden Petera} Waterhouse'a (2016)

Abstrakt: Artykuł dotyczy eksperymentu prozatorskiego Die Auswandernden austriackiego pisarza Petera Waterhouse'a i ilustratorki Nanne Meyer z 2016 roku. Książka zaliczana jest do kategorii tekstów literackich odzwierciedlających doświadczenia uchodźców i osób ubiegających się o status uchodźcy, które pojawiają się na niemieckim rynku wydawniczym od 2012 roku. Artykuł ma na celu opisanie strategii literackich stosowanych przez Waterhouse'a do przedstawienia sytuacji uchodźców. Bada ukryte wypowiedzi programowe autora, konstrukcję bohaterki jako postaci uchodźczyni i ubiegającej się o azyl oraz refleksje Waterhouse'a nad językiem, zwłaszcza językiem austriackiego sądownictwa.

${ }^{1}$ Angesichts der poetologischen Aussagen Waterhouses über das Verhältnis von „Erzählen“ und „Nicht-Erzählen“ und des komplexen Umgangs des Autors mit diesen Kategorien in Den Auswandernden erscheinen weder der Begriff „Flüchtlingsnarrativ“ noch der von Agnes Woolley vorgeschlagene Begriff ,refugee and asylum-seeker narrative“ ohne Weiteres auf das Buch anwendbar. Vgl. A. Woolley, Contemporary Asylum Narratives. Representing Refugees in the Twenty-First Century, London 2015, S. 68. 
Słowa kluczowe: Peter Waterhouse, Nanne Meyer, migracja jako temat literacki, współczesna literatura austriacka, refleksja nad językiem, język prawniczy, eksperyment prozatorski

Schlüsselwörter: Peter Waterhouse, Nanne Meyer, Flucht und Asylsuche als literarische Themen, österreichische Gegenwartsliteratur, Sprachreflexion, Auseinandersetzung mit der Sprache des Justizsystems, Prosaexperiment

$* * *$

„Und so will jedes Wort wörtlich genommen werden, sonst verwest es zur Lüge (...).“2

Das im Zentrum des vorliegenden Beitrags stehende Prosaexperiment Die Auswandernden, das kurz nach dem Erscheinen auf die Shortlist für den Österreichischen Buchpreis gesetzt wurde, gehört in die Reihe literarischer Repräsentationen der Flüchtlingsthematik, die seit 2012 auf dem deutschsprachigen Buchmarkt erschienen. ${ }^{3}$ Der Autor des Textes, der 1956 als Sohn eines britischen Offiziers und einer Österreicherin geborene, bilingual erzogene Schriftsteller und Übersetzer Peter Waterhouse gilt als eine der ,profiliertesten Stimmen der deutschsprachigen literarischen Avantgarde". ${ }^{4}$ Gedichte, Prosa und Essays des Autors, der 1984 an der Universität Wien mit einer Arbeit über Paul Celan promovierte, zeichnen sich durch ein hohes $\mathrm{Ma} ß$ an Ambiguität und Autoreflexivität aus und erkunden fortwährend, wie sich die Sprache und Wahrnehmung gegenseitig beeinflussen. ${ }^{5}$ In Den Auswandernden rekurrierte Waterhouse auf charakteristische Verfahren seiner früheren Texte, wobei er sich einer aktuellen Thematik zuwandte: Sein Buch erschien auf dem Buchmarkt im Schatten der 2015 erfolgten (mit dem kontrovers diskutierten Begriff der „Flüchtlingskrise“ bezeichneten), Kulmination globaler Fluchtbewegungen, in deren Folge 1,25 Millionen Menschen in Europa nach Asyl suchten. Das Prosaexperiment war allerdings keine rasche Reaktion des Autors auf diese dramatischen Entwicklungen: In diesem Werk werden Erfahrungen des

${ }^{2}$ R. Musil, Der Mann ohne Eigenschaften, Reinbeck bei Hamburg 1978, S. 749.

${ }^{3}$ Eine synthetische Übersicht jener ,écriture (...) engagée sur un sujet d'une grande actualité“ bieten: von Emmanuelle Terrones: E. Terrones, „Flüchtlingsromane“: une nouvelle catégorie littéraire?, https://migrexil.hypotheses.org/250\#_ftn13 (Abruf: 15.04.2021). Th. Hardtke, J. Kleine, Ch. Payne, Niemandsbuchten und Schutzbefohlene. Flucht-Räume und Flüchtlingsfiguren in der deutschsprachigen Gegenwartsliteratur [in:] Niemandsbuchten und Schutzbefohlene. Flucht-Räume und Flüchtlingsfiguren in der deutschsprachigen Gegenwartsliteratur, hrsg. v. Th. Hardke, J. Kleine, Ch. Payne, Göttingen 2017, S. 9-22.

${ }^{4}$ E. De Felip, Inwendige Landschaften oder Die leeren Räume der Sprache Peter Waterhouse' „,Spaziergang als Himmelskunst“ und Oswald Eggers „Im Anger des Achilles“ Möglichkeiten und Grenzen einer wissenschaftlichen Lektüre, „Studia Austriaca“ 2013, XXI, S. 51-80, hier S. 51.

${ }^{5}$ Vgl. I. Noël, Peter Waterhouse. Das lyrische Werk [in:] Kindler Kompakt. Deutsche Literatur der Gegenwart, hrsg. v. Ch. Freudenstein-Arnold, Stuttgart 2015, S. 29-37, hier S. 29-30.

Waterhouses Schaffen wurde im Zeitraum 1989-2018 mit 18 deutschen und österreichischen Preisen, darunter Christine Lavant-Lyrikpreis (1994), Adolf-Mejstrik-Ehrengabe für Lyrik der Deutschen Schillerstiftung (2000), H.C. Artmann-Preis (2004), Erich-Fried-Preis (2004), Ernst-JandlPreis (2011) gewürdigt. 2012 wurde dem Autor Großer Österreichischer Staatspreis verliehen. 
Autors verarbeitet, der sich seit dem Anfang des Dezenniums für die Asylbewerber in Österreich politisch einsetzte. ${ }^{6}$

Neben den Namen Waterhouses nennt der Einband den Namen der bildenden Künstlerin Nanne Meyer. Die in Berlin lebende Co-Autorin hat die Publikation mit 58 doppelseitigen, mit abstrakten Elementen operierenden Zeichnungen ausgestattet, die auf Motive der Flucht und Asylsuche eingehen und partiell als Ergänzungen und partiell als visuelle Äquivalente der sprachlichen Aussagen des Schriftstellers fungieren. Der Anteil Meyers an dem Buch und die intermedialen Bezüge zwischen dessen sprachlichen und bildlichen Elementen müssen im vorliegenden Beitrag außer Acht gelassen werden, da eine eingehende Auseinandersetzung mit diesem Thema den hier zur Verfügung stehenden Rahmen sprengen würde. Der Beitrag setzt sich indes zum Ziel, die Strategien zu beschreiben, mit denen Waterhouse das Thema der Flucht und Asylsuche verhandelt. Der Artikel erkundet zunächst die poetologischen Selbstauskünfte des Autors, um danach der Konstruktion der Protagonistin als einer Flüchtlingsfigur sowie die Auseinandersetzung mit der Thematik des Spracherwerbs und der Sprache der Justiz in den Auswandernden nachzugehen.

\section{Poetologische Verortungen}

Grundlegend für die Lektüre des Buches erscheint die Heranziehen seiner poetologischen Prämissen. Wie bei allen anderen durch narrative Komplexität und sprachliche Dichte gekennzeichneten Texten Waterhouses bleibt auch im Falle Der Auswandernden eine Gattungszuordnung problematisch. Eleonore de Felip nennt das Buch ,a novel with no real plot" ${ }^{67}$, Petra Lohrmann konstatiert, Die Auswandernden seien ,definitiv kein Roman, (...) keine Erzählung, kein Erfahrungsbericht, keine Dokumentation. Am ehesten ist es ein langes Gedicht $(\ldots)^{\text {‘68 }}$ Waterhouse selbst situiert sein Werk in der Nähe eines Traktats, indem er zum Schluss des Textes folgende Passage aus Benjamins kryptischer „Erkenntniskritischer Vorrede" zum Trauerspielbuch zitiert:

Darstellung als Umweg - das ist denn der methodische Charakter des Traktats. Verzicht auf den unabgesetzten Lauf der Intention ist sein erstes Kennzeichen. Ausdauernd hebt das Denken stets von neuem an, umständlich geht es auf die Sache selbst zurück. Dieses un-

${ }^{6} 2012$ trat Waterhouse mit der Rede Gesetz und Entsetzen, in der er seine Empörung über die Situation der Flüchtlinge in Österreich äußerte, im Asylgerichtshof in Wien auf. 2013 verbrachte er mehrere Nachtstunden in der Wiener Votivkirche, um seine Solidarität mit den dort protestierenden Asylbewerbern zum Ausdruck zu bringen. Vgl. E. De Felip, 'Will you grant me grace?': Peter Waterhouse's Poetics of Possible Worlds in „Die Auswandernden” (2016), „Austrian Studies“2018, Vol. 128, S. 124-138, S. 124.

7 Ibid., S. 136.

${ }^{8}$ P. Lohrman, Peter Waterhouse: Die Auswandernden, https://www.gute-literatur-meineempfehlung.de/autoren-m-z/waterhouse-peter/peter-waterhouse-die-auswandernden/\#: :text=Es\%20 ist $\% 20$ definitiv $\% 20$ kein $\% 20$ Roman,selbst $\% 2 \mathrm{C} \% 20$ es $\% 20$ ist $\% 20$ ein $\% 20$ Traktat.\&text $=$ Sein $\% 20$ Traktat $\% 20$ ist $\% 20$ ein\%20unglaubliche,mit $\% 20$ dem\%20Innersten\%20des\%20Menschen (Abruf: 20.04.2021). 
ablässige Atemholen ist die eigenste Daseinsform der Kontemplation. Denn indem sie den unterschiedlichen Sinnstufen bei der Betrachtung eines und desselben Gegenstandes folgt, empfängt sie den Antrieb ihres stets erneuten Einsetzens ebenso wie die Rechtfertigung ihrer intermittierenden Rhythmik. ${ }^{9}$

Mit den in poetologischer Funktion angeführten und als ,die fünf schönsten der Welt" (A 248) bezeichneten Sätzen Benjamins stellt der Verfasser den Erkenntniswert seines Werks heraus. Bei der Wiedergabe der Schicksals der Protagonistin verzichtet er auf Linearität, entlegene Passagen des Buches bleiben mehrfach durch Rekonfigurationen des Materials und seine Anverwandlungen verbunden, wobei Vernetzungen entstehen, die den Text als Gewebe herausstellen. Wie in anderen Schreibprojekten des Autors dominiert auch hier die Frageform. Der charakteristische Frageduktus ${ }^{10}$ ist eine Herausforderung für die Leser, doch diese Strategie erlaubt dem Autor, das Benjaminsche Postulat nach dem fortwährenden Neuanheben des Denkens zu realisieren. Dabei demonstriert der Schriftsteller, das der durch beständiges Befragen der gerade gewonnenen Erkenntnisse erlangte Neuanfang nicht zu Antworten, sondern zu neuen Fragen führt.

Als eine weitere poetologische Selbstauskunft des Autors ist m. E. sein Kommentar zu Adalbert Stifters Turmalin anzuerkennen. Bei einem Versuch, die literarischen Strategien dieser Erzählung zu charakterisieren, konstatiert Waterhouse: „Stifters Erzählung erzählte und sie erzählte nicht“ (A 23)11. Durch den Kontext wird der Satz als affirmativ ausgewiesen, er ergänzt Waterhouses Bekenntnis zu einer epischen Form, die eine fragmentarische Handlungsstruktur auszeichnet und die die konventionellen Zusammenhänge zwischen Wort und Sache, Name und Merkmal infrage stellt. ${ }^{12}$ Mit ihm scheint eine andere Passage zusammenzuhängen, an der Waterhouse die Epik, bzw. den für diese grundlegenden Gestus des Geschichtenerzählens mit folgenden Worten seiner Protagonistin thematisiert:

Wenn sie eine Erzählung lese, wolle sie sie vielleicht ein zweites Mal lesen und dann ein drittes. Selten ein viertes Mal. (...) Vielleicht ein paar Jahre später mit großem Interesse (...), von Neuigkeiten nicht erneuert, und schließlich ohne den Wunsch, die Erzählung noch einmal zur Hand zu nehmen, und es blieben nur wenige Satze im Gedächtnis. (...) Eine Erzählung kann die Wahrheit nicht finden (...). Das kurze Gedicht findet sie. (...) Wo nichts passiert, kann ich acht geben. Wenn ich achtgebe, betrachte ich sie. (...) In einem Gedicht sprechen die Worte langsam, als ob sie sprechen lernten. (A 93f.)

Das auf schlichte Wiedergabe von Ereignissen reduzierte Erzählen wird von Waterhouse deswegen als problematisch definiert, weil es den erzählten Inhalt

${ }^{9}$ Das Zitat aus Benjamins Ursprung des deutschen Trauerspiels wird hier nach Waterhouse wiedergegeben. P. Waterhouse, N. Meyer, Die Auswandernden, Fürth 2016, S. 248. (Die Zitate aus dieser Ausgabe werden im Folgenden im laufenden Text mit der Sigle A und der einschlägigen Seitennummer angegeben.)

${ }^{10}$ Vgl. Ch. Ivanovič, Vorwort, [in:] Darstellung als Umweg. Essays und Materialien zu (Krieg und Welt) von Peter Waterhouse, hrsg. v. Ch. Ivanovič Wien 2020, S. 11-23, hier S. 16.

${ }^{11}$ Auch die von Waterhouse reflektierte Strategie der Auslassungen (vgl. A 24) kann in Zusammenhang mit seinem eigenen Prosaexperiment gebracht werden.

${ }_{12}$ Vgl. T. Albrecht, Turmalin [in:] Adalbert Stifter Handbuch, hrsg. v. Ch. Begemann, D. Giuriato, Stuttgart 2017, S. 87-91, hier S. 90. 
schnell vergessen lässt. Dies widerspricht dem vom Schriftsteller durch die Referenz auf das Traktat definierten Ziel seines Schreibens, das die Leser mit seinem durch stete Fragen beunruhigenden (und wie von Benjamin postuliert, oft umständlichen) Duktus zum Denken inspirieren möchte. Der kritisierten narrativen Form stellt Waterhouse einen erschriebenen Raum entgegen, der durch Sprachreflexion definiert wird. Diese Definition wird nicht nur dem Konzept des Autors von Lyrik und seinen eigenen lyrischen Texten gerecht, sondern scheint auch seinem Begriff von Literatur schlechthin zu entsprechen und kann somit auf Die Auswandernden bezogen werden.

In dem Buch, in dessen Zentrum eine Flüchtlingsfigur steht, bekommt die Absage an das als Aneinanderreihung von Begebenheiten verstandene Erzählen eine besondere Bedeutung. Mit ihr scheint Waterhouse mitzuteilen, dass er nicht der Lust jener Leser entsprechen möchte, die ihren Voyeurismus an Leidnarrativen befriedigen, wonach sie das Erzählte bald dem Vergessen anheimfallen lassen. Indem er die Worte „langsam sprechen“13 und seine Erzählung immer wieder neu anheben lässt, verlangt der Schriftsteller eine achtsame Lektüre, die sich nicht zuletzt auch der Herausforderung zu stellen hat, die bruchstückhaft vermittelte Geschichte der Protagonistin zu entschlüsseln.

\section{Die Auswandernde: Media}

Im Zentrum Der Auswandernden steht die Geschichte der Asylbewerberin Media, die mit ihrer achtjährigen Tochter aus dem Kaukasus nach Österreich geflohen ist. Ihre Versuche, in Wien anzukommen, ihre Wanderungen durch diese Stadt, ihre mühsamen Behördenwege und ihr Weg zur deutschen Sprache werden von einem anonymen Ich-Erzähler geschildert, dessen Biografie Affinitäten zum Lebenslauf Waterhouses aufweist. ${ }^{14}$

Die Schwierigkeiten bei der Erschließung von Medias Schicksalen bei der Lektüre des Buches ergeben sich aus der spezifischen Verfasstheit des Textes, den Eleonore De Felip folgendermaßen beschreibt: „The text presents itself as a complex interweaving of thoughts, perceptions and memories, hence there is also no chronology of events, but multiple levels which coexist simultaneously“" ${ }^{15}$

Die verstreuten Informationen zu Medias Vergangenheit und Gegenwart nehmen im so konstruierten Text deutlich weniger Platz als Reflexionen über die Flucht, Sprache und Recht ein. Darüber hinaus bleibt die Geschichte der Protagonistin voller Leerstellen. Weitgehend ausgespart wurden u.a. genauere Angaben über die Gründe, die Media nach Wien geführt haben. Evident wird, dass sie in ihrem Heimatland Opfer eines Angriffs wurde, der Spuren an ihren Hand- und

${ }^{13}$ Vgl. einen weiteren poetologischen Satz der Der Auswandernden: „In einem Gedicht sprechen die Worte langsam, als ob sie sprechen lernten“. (A 93f.)

${ }^{14}$ Vgl. I. Noël, Übersetzen anstatt unterscheiden. 'Die Auswandernden 'von Peter Waterhouse und Nanne Meyer: ein Plädoyer mit poetischen Mitteln, https://literaturkritik.de/uebersetzen-anstattunterscheiden-auswandernden-peter-waterhouse-nanne-meyer-ein-plaedoyer-mit-poetischenmitteln,23207.html (Abruf: 24.06.2021).

${ }^{15}$ E. De Felip, 'Will you grant me grace?': Peter Waterhouse's Poetics..., op. cit., S. 136. 
Fußgelenken hinterlassen hat. Waterhouse entspricht seinen poetologischen Prämissen, indem er nur knapp auf die Leiderfahrungen der Protagonistin eingeht. Die Angreifer bleiben unbekannt, fest steht nur, dass Media ihr Land fluchtartig verlassen musste. Der Erzähler teilt mit, dass sie und ihr Freund sich bei der Suche nach einem Zufluchtsort gegen Deutschland entscheiden, weil die ,die Geheimdienste beider Länder eine Zusammenarbeit verband“ (A 25). Die Angabe wird verständlich dank der voranstehenden Information, dass es sich bei dem Freund, um einen „Mitarbeiter der Omega“"16 handelt. Es erscheint kennzeichnend, dass die Figur des Freundes nach dieser einmaligen lapidaren Auskunft im Buch nicht mehr erwähnt wird.

Das Herkunftsland Medias wird im Text nie direkt genannt, dass es sich dabei um Georgien handelt, verraten (neben des bereits erwähnten vieldeutigen Namen „Omega“), der von ihr ausgesprochene, ins lateinische Alphabet transkribierte Satz (A 123), ein im georgischen Alphabet wiedergegebene Ausdruck aus ihrem Wörterbuch (A 117) und die von ihr zitierten Märchenanfänge. Spärlich verfährt der Autor mit den Angaben zu Medias Tochter, deren Vater wird nicht einmal benannt. Die Darstellung von Medias Aufenthalt in Wien und dem Scheitern ihres Asylgesuchs wird um punktuell wiedergegebene Ereignisse und Szenen zentriert, die selbst bei aufmerksamer Lektüre nur eine fragmentarische, bruchstückhafte Geschichte ergeben.

Als den markantesten Aspekt der literarischen Texte, die sich der Flüchtlingsproblematik widmen, bezeichnete Emanuelle Terrones, dass sie häufig mit dem Konzept des Kollektivs operieren, das die Opposition sie/wir und damit auch den Konflikt der Kulturen deutlich macht. ${ }^{17}$ Dieser Form der Narrativierung von Flüchtlingsschicksalen stellt sich Waterhouse entgegen, indem er sich einem Einzelschicksal zuwendet. Vor der Folie seiner sprachanalytisch orientierten Rede Gesetz und Entsetzen wird ersichtlich, welch wichtige Bedeutung der Ausbildung der Protagonistin zukommt: In dieser Rede richtete sich der Schriftsteller u.a. gegen die im Asylverfahren verankerte diskriminierenden Negation der intellektuellen Kompetenzen der Flüchtlinge, die manchmal vier oder fünf Sprachen sprechen und die dazugehörigen Buchstabiersysteme kennen, doch in dem Asylland als „Analphabeten“ wahrgenommen werden, weil sie seines Alphabets und seiner Sprache nicht mächtig sind. ${ }^{18}$ In Den Auswandernden konstruiert der Autor eine Protagonistin, die nach Wien aus einem kaukasischen Dorf gekommen ist. Allerdings entzieht sie sich stereotypen Zuschreibungen, die sie auf diesen Herkunftsort festlegen würden. Fest steht, dass Media drei Schriftsysteme kennt, eine Akademikerin ist und sich in ihrem Studium mit philosophischen Texten zur Ethik auseinandergesetzt hat. Ihre sprachreflexiven Fragen und tiefgründige Kommentare lassen den Erzähler seine eigene Welt und Sprache mit neuen Augen sehen.

${ }^{16}$ Der vieldeutige Eigenname „Omega“ wird im Text nicht erklärt, kann aber durch Recherche als der Name einer Spezialeinheit georgischen Geheimdienstes identifiziert werden.

17 E. Terrones, op. cit.

18 P. Waterhouse, Gesetz und Entsetzen, https://www.youtube.com/watch?v=Fa9fdLUhxa8 (Abruf: 25.05.2021). Hier vgl. Minuten 11.05-16.14. 


\section{Worte, die langsam sprechen, als ob sie sprechen lernten}

Das poetologische Programm eines literarischen Textes, in dem die Worte ,langsam sprechen, als ob sie sprechen lernten" (vgl. Anm. 13) realisieren Die Auswandernden zunächst, indem sie den Erzähler Media beim Erlernen des Deutschen begleiten lassen, womit Waterhouse das Motiv des Spracherwerbs variiert, das er 2006 in seinem autobiografischen Buch Krieg und die Welt sprachreflexiv funktionalisiert hat. ${ }^{19}$ Nach dem lange zurückliegenden Schulunterricht spricht Media bei ihrer Ankunft in Wien kaum Deutsch. Ihre Arbeit an dieser Sprache bleibt nicht ohne komische Effekte, die sich aus Abweichungen von lexikalischen Standards ergeben.

Sie [Media] fragte nicht nach der Bedeutung eines Worts, sondern sprach ein Wort auf eine neue Weise - und erneuerte es vielleicht. Sie nannte das Buch von Astrid Lindgren Pippi Langstrumpfhose. Sie schrieb in einem Bewerbungsbrief das Wort Timm. Auf meine Frage, was ein Timm sein, sagte sie, das wisse sie nicht, Timm, sie habe das Wort gehört. Oder Tims vielleicht. Man habe ihr gesagt, sie werde in einem Timm arbeiten. Ein gutes Timm, wenn Menschen gut zueinander passen. Wir lachten oft über die Wörter, wir lachten über Pippi Langstrumpfhose, die Strumpfhose war neu, noch neuer als Astrid Lindgrens Roman. (A 23, Hervorhebungen im Original)

Der Erzähler verbleibt nicht bei den Verweisen auf den spielerisch-kreativen Aspekt des Spracherwerbs. In dieser wie auch in zahlreichen anderen Passagen des Buches betont er, dass Medias Verstöße gegen die Normen der deutschen Sprache, ihre Wortwahl, Suche nach den benachbarten Ausdrücken und die daraus entstehenden Verfremdungen der Wörter, ihre Fragen und überraschende Bemerkungen ihn selbst nach neuen Bedeutungen und Zusammenhängen zwischen den Wörtern und Erscheinungen suchen lassen. Es sind Medias Fragen, die den Erzähler die Logik deutscher Ausdrücke anzweifeln lassen. Sie fragt z.B. „Was bedeutet gescheitert? Was ist ein gescheiterter Räuber? (...) Hat er nichts geräubert und ist er kein Räuber? (A 26)“ Sie lassen ihn darüber hinaus immer wieder nach Synonymen suchen, legen die vergessenen Vernetzungen zwischen den Wörtern offen und inspirieren zu weiteren Reflexionen. Ein sinnfälliges Beispiel liefern hierbei die Versuche der Protagonistin, die Bezüge zwischen ,lang“, „langweilen“, ,verlangen“ und ,verlängern“ (A 53-55) zu ermitteln, was den Erzähler in Gedanken den verschlungenen Zusammenhängen zwischen den Worten und Phänomenen „Begehren“, „Zeit" und „Verzögerung“ nachgehen lässt.

Waterhouse stellt Medias kreativen, aufmerksamen Umgang mit den Wörtern, der laut seinem Urteil sowohl den kindlichen Spracherwerb als auch den Fremdsprachenerwerb charakterisiert, auf eine Stufe mit dem Schreiben von den von ihm als „Gedichte“ ausgewiesenen literarischen Texten, jenen also, in deren Zentrum die Sprache steht. Er schreibt:

Wer eine Sprache zu sprechen beginnt, sie Tag für Tag zu sprechen beginnt und wiederholt, spricht ein (...) Gedicht. (...) Am schönsten sind die kurzen Gedichte, in welchen ein Wort

${ }_{19}$ Vgl. Ch. Ivanovič, op. cit., S. 16. 
nicht zu verstehen ist. In einem kurzen Gedicht kann ich auf alles achtgeben. Wenn ich eine Sprache lerne, ist sie ein kurzes Gedicht. (A 94)

Dieser behutsame, fragende Umgang mit der Sprache bestimmt die Auseinandersetzung des Erzählers mit dem Wortschatz der österreichischen Asylgesetze, Haftbefehle und Protokolle der Asylbehörden. Mit Emanuelle Terrones ist festzustellen, dass Kritik an der Justiz eine Konstante der literarischen Repräsentationen von Flüchtlingserfahrungen darstellt, wobei zahlreiche Passagen die Absurdität staatlicher Regeln und Prinzipien anprangern. ${ }^{20}$ Als ein charakteristisches Merkmal von Waterhouses Buch ist zu erkennen, dass es nur knapp auf die Realien der Einvernahmen eingeht. Einblicke in das System des Asylrechts gewährt er den Lesern vor allem durch Verweise auf die sprachliche Verfasstheit der einschlägigen Texte. Wie in seiner Rede Gesetz und Entsetzen hinterfragt Waterhouse auch in Die Auswandernden die juristischen Formulierungen als ungerechtfertigt und prangert die damit verbundenen Konzepte an. Er stellt die juristischen Begriffe infrage, indem er ihre Konnotationen reflektiert. Dabei stellt er die semantischen Ambivalenzen der Sprache heraus, z.B. indem er auf die Unterschiede zwischen der Alltagssprache und der Amtssprache folgendermaßen eingeht:

In Protokollen und Gerichtsbeschlüssen las ich das Wort betreten in seiner alten Bedeutung, die in der gegenwärtigen Sprache längst nicht mehr galt. Im Haftbefehl las ich: Sie wurden am 14. Februar um 14.30 Uhr auf dem Roosevelt Platz 3 in Wien 9. betreten. (...) und ich korrigierte den Satz: Sie haben den Roosevelt Platz betreten; und das Haus Roosevelt Platz betreten. Eintreten in das Haus oder das Haus betreten? (...) War das Betreten ein immerzu verbotener Schritt? Hörte nicht, wer das Wort betreten hörte, schon das Wort verboten? (...) Hörte Herr Magister Fremdenpolizist, indem er in den Bescheid schrieb: Sie wurden auf dem Roosevelt Platz betreten, hörte er ebenso wie ich: Sie ... betreten verboten ... Sie $z u$ betreten ... ist verboten. (A $87 \mathrm{f}$. Hervorhebungen im Original)

Der Erzähler analysiert die semantischen Differenzen des Homonyms ,,betreten“, dem in der Alltagssprache die Bedeutung, ,in einen Raum hineingehen“, ,seinen Fuß auf etwas setzen" zukommt, während er in der (in diesem Fall, wie vom Autor angedeutet, auf das Mittelhochdeutsche rekurrierenden) österreichischen Amtssprache als ein Synonym des Verbs „,ergreifen“ funktioniert. Überdies wird die zuerst genannte Bedeutung des Verbs mit dem semantischen Feld des Verbots in Verbindung gebracht, womit eine Konstante des sprachlichen Usus identifiziert wird. Die Herausstellung dieser Zusammenhänge macht sowohl auf den politischen Diskurs um das Phänomen der Flucht als auch auf die außersprachliche Wirklichkeit, d.i. die Situation der Flüchtlinge aufmerksam, die sofort nach der Ankunft in dem Asylland, mit dem sie Hoffnungen auf einen Neuanfang verbinden, von dessen Behörden ,ergriffen“, d.h. als potenzielle Kriminelle behandelt werden.

Dieser Einstellung, die der Autor an Ausdrücken aus der juristischen Sprache festmacht, wird eine Haltung entgegengestellt, die Waterhouse mit dem englischen Wort ,grace“ identifiziert. Den Ausdruck, ,Will you grant me just a few minutes grace“" situiert der Erzähler vorerst in seiner Erfahrung. Er hört ihn in einem

\footnotetext{
${ }^{20}$ E. Terrones, op. cit.
} 
Film über die Vorbereitung des Musicals Madame Butterfly, in dem sich einer der Sänger mit diesem Satz kurz vor der Premiere an die Regisseure wandte. Die Äußerung bewegt den Erzähler zum Nachdenken über den vieldeutigen Begriff:

Ich wusste nicht sofort, worum der Sänger gebeten hatte. Hatte er gebeten um Anmut, um Dankbarkeit, um einen schönen Anblick, einen Aspekt, ein Ornament? Aber das Wort beschrieb (...) mich. Es beschrieb lange Zeit. (...) Es war nicht zu übersetzen und es bedeutete nicht Zeit, Entlastung, Verzögerung. Es war eines der schönsten Wörter der Welt. (...) Grace es bedeutete nicht Aufschub. Nicht die Entlastung von einer Aufgabe. Nicht Immunität vor einer Strafe. Nicht Unerreichbarkeit für Forderungen und Ansprüche. Sondern alle Schönheiten der Welt. Die ganze Dankbarkeit. (A 91 f. Hervorhebungen im Original)

In der Extrapolation dieser situationsgebundenen Äußerung sieht der Erzähler eine Möglichkeit, die Lage der Asylsuchenden angemessen zu beschreiben:

Die vielen, die aus so vielen Ländern nach Österreich und nach Wien und nach Europa flüchteten, sie suchten nicht an um Asyl (...) Sie baten um grace, also nicht um einen Zeitraum, nicht um die Immunität, sondern um die Schönheit der Welt. Um Gunst; um Freundschaft; um Liebe; um Wohlwollen. Will you grant me grace? Lebensfreude? (A 92, Hervorhebungen im Original)

In den früheren Passagen seines Textes reflektierte Waterhouse Beispiele von juristischen Ausdrücken, um vorzuführen, wie die scheinbar neutrale Rechtssprache die Wahrnehmung des Anderen und folglich auch Wirklichkeit negativ beeinflusst. Dabei verzichtete er beim Thematisieren der Flucht-und Asylerfahrung auf die Entfaltung von Leidnarrativen, die, nachdem sie die emotionalen Bedürfnisse der Leser befriedigt haben, schnell vergessen werden. Statt dessen berief sich der Autor auf die erkenntnisfördernde Funktion der Literatur, die auf einem behutsamen, sprachreflexiven Umgang mit dem Wort aufbaut.

Vor dieser Folie kommt der an der oben zitierten Stelle erfolgten Zuwendung zu dem im ethischen, juristischen und ästhetischen Diskurs verankerten englischen Ausdruck grace, (dem auf Deutsch u.a. nicht nur die Wörter „Wohlwollen“, „Gnade“, „Aufschub“, sondern auch „Anmut“ entsprechen) und der Wiederholung der Frage Will you grant me grace unter Angabe von mehreren ihrer deutschen Äquivalente eine zentrale Rolle in Den Auswandernden zu. Nicht nur situiert der Autor mit ihr das Denken über die Flucht und Asylsuche jenseits gängiger politischer, sozialer und ökonomischer Überlegungen, womit er einen geradezu utopischen Horizont aufreißt. Die Reflexion über den mehrdeutigen englischen Ausdruck bedeutet eine Einladung, über die dem Asylantrag innewohnende Bitte um Gastfreundschaft und Solidarität und damit auch über die Beziehung zwischen sich selbst und dem Anderen, nachzudenken. Waterhouses Text überlässt es hierbei den Lesern, den Raum zwischen dem Unmöglichen und Möglichen selbst zu ergründen.

\section{Bibliografie (References)}

Albrecht T., Turmalin [in:] Adalbert Stifter Handbuch, hrsg. v. Ch. Begemann, D. Giuriato, Stuttgart 2017, S. 87-91. 
Brüggemeyer M., Peter Waterhouse: 'Die Auswandernden'. Poetische Perspektive auf eines der größten Themen der Gegenwart, https://www.deutschlandfunk.de/peterwaterhouse-die-auswandernden-poetische-perspektive.700.de.html?dram:article $\mathrm{id}=375007$ (Abruf: 25.04.2021).

De Felip E., Inwendige Landschaften oder Die leeren Räume der Sprache. Peter Waterhouse, ,Spaziergang als Himmelskunst“ und Oswald Eggers „,Im Anger des Achilles“. Möglichkeiten und Grenzen einer wissenschaftlichen Lektüre, „Studia Austriaca“ 2013, XXI, S. 51-80.

De Felip E., 'Will you grant me grace?': Peter Waterhouse's Poetics of Possible Worlds in „Die Auswandernden” (2016), „Austrian Studies“ 2018, Vol. 128, S. 124-138.

Fasthuber S., Wer „, betreten“ sagt, muss auch ,verboten “ sagen. Falter 06/17 vom 8.02.2017, https:/www.falter.at/zeitung/20170208/wer-betreten-sagt-muss-auchverboten-sagen/11 f00bdf4b?ver=a (Abruf: 10.09.2020).

Hardtke Th., Kleine J., Payne Ch., Niemandsbuchten und Schutzbefohlene. FluchtRäume und Flüchtlingsfiguren in der deutschsprachigen Gegenwartsliteratur [in:] Niemandsbuchten und Schutzbefohlene. Flucht-Räume und Flüchtlingsfiguren in der deutschsprachigen Gegenwartsliteratur, hrsg. v. Th. Hardke, J. Kleine, Ch. Payne, Göttingen 2017, S. 9-22.

Ch. Ivanovič, Vorwort, [in:] Darstellung als Umweg. Essays und Materialien zu (Krieg und Welt) von Peter Waterhouse, hrsg. v. Ch. Ivanovič Wien 2020, S. 11-23,

Lohrman P., Peter Waterhouse: Die Auswandernden, https://www.gute-literatur-meine-empfehlung.de/autoren-m-z/waterhouse-peter/peter-waterhouse-die-auswandernden/\#: : text=Es\%20ist $\% 20$ definitiv $\% 20 \mathrm{kein} \% 20$ Roman,selbst\%2C\%20es\%20ist\%20ein\%20Traktat.\&text=Sein $\% 20$ Traktat $\% 20$ ist $\% 20$ ein $\% 20$ unglaubliche,mit $\% 20$ dem $\% 20$ Innersten $\% 20 \mathrm{des} \% 20$ Menschen (Abruf: 20.04.2021).

Musil R., Der Mann ohne Eigenschaften, Reinbeck bei Hamburg 1978.

Nischkauer A., Peter Waterhouse/Nanne Meyer: Die Auswandernden, „Wespennest“, 172/217, S. 107-110.

Noël I., Peter Waterhouse. Das lyrische Werk [in:] Kindler Kompakt. Deutsche Literatur der Gegenwart, hrsg. v. Ch. Freudenstein-Arnold,Stuttgart 2015, S. 29-37.

Noël I., Übersetzen anstatt unterscheiden. 'Die Auswandernden' von Peter Waterhouse und Nanne Meyer: ein Plädoyer mit poetischen Mitteln, https://literaturkritik.de/uebersetzen-anstatt-unterscheiden-auswandernden-peter-waterhouse-nanne-meyer-ein-plaedoyer-mit-poetischen-mitteln,23207.html (Abruf: 24.06.2021).

Terrones E., „Flüchtlingsromane“: une nouvelle catégorie littéraire?, https://migrexil.hypotheses.org/250\#ftn13 (Abruf: 15.04.2021).

Woolley A., Contemporary Asylum Narratives. Representing Refugees in the TwentyFirst Century, London 2015.

Waterhouse P., Meyer N., Die Auswandernden, Fürth 2016.

Waterhouse P., Gesetz und Entsetzen, https://www.youtube.com/watch?v= Fa9fdLUhxa8 (Abruf: 25.05.2021). 